\title{
Immunosuppressive effect of bladder cancer on function of dendritic cells involving of Jak2/STAT3 pathway
}

\author{
Weigang Xiu' ${ }^{1,2}$, Juan Ma ${ }^{1,2}$, Ting Lei ${ }^{1,2}$, Man Zhang ${ }^{1,2}$, Shangyan Zhou ${ }^{1,2}$ \\ ${ }^{1}$ Clinical Laboratory Medicine, Beijing Shijitan Hospital, Capital Medical University, Beijing, China \\ ${ }^{2}$ Beijing Key Laboratory of Urinary Cellular Molecular Diagnostics, Beijing, China \\ Correspondence to: Man Zhang, email: mzhang99@aliyun.com \\ Keywords: bladder cancer, dendritic cell, anti-tumor immunity, immunotherapy, janus kinase 2/signal transducer and \\ activator of transcription 3 pathway \\ Received: April 20, $2016 \quad$ Accepted: August 11, $2016 \quad$ Published: August 20, 2016
}

ABSTRACT

Function of dendritic cells (DCs) is impaired by some cancer cells. However, the effect of bladder cancer cell (BCC) on phenotype and function of DCs remains unclear. In this study, healthy human peripheral blood mononuclear cells (PBMCs) derived DCs were co-cultured with BCC pumc-91 and adriamycin-resistant pumc-91/ADM. The expression of DC markers and costimulatory molecules decreased after co-culture. Co-cultured DCs rapidly underwent apoptosis, and had a declined capability to produce IL-8 and RANTES. Furthermore, co-cultured DCs showed impaired allogeneic $T$ cell proliferation and T cell-derived cytokine secretion. Finally, AG490, a Jak2/STAT3 inhibitor, restored the expression of DC markers and costimulatory molecules. Of note, compared with control DCs, DCs co-cultured with pumc-91 produced more IP-10; DCs co-cultured with pumc-91/ADM secreted more MIG. Taken together, these results suggest BCC may inhibit maturation and function of DCs involving of Jak2/STAT3 pathway, and there may be different mechanisms by which adriamycin-resistant BCC restrains DC function in antitumor immune response.

\section{INTRODUCTION}

Bladder cancer is one of the most common malignancies of urinary system, about 429800 new cases and 165100 deaths occurring in 2012 worldwide [1]. Bladder cancer can be categorized as non-muscle invasive bladder cancer (NMIBC) and muscleinvasive bladder cancer (MIBC). The characteristic of non-muscle invasive bladder cancer is frequent recurrence and low mortality. Moreover, $10-20 \%$ of non-muscle invasive bladder cancer will progress to muscle-invasive bladder cancer [2]. In the case of muscle-invasive bladder cancer, despite exist multiple treatment strategies, such as radical cystectomy, radiation therapy and chemotherapy, survival rates are poor [3]. These therapies restrict growth and development of tumor, however, they do not prevent recurrence and drug-resistant [4, 5]. Therefore, effective strategies are needed urgently.

Dendritic cells (DCs) are the most powerful antigen-presenting cells (APCs). To induce efficient lymphocyte response, antigen is bound to major histocompatibility complex (MHC) of DCs, then presented to and identified by $\mathrm{T}$ cells. In addition, DC vaccines highly express CD86, CD80, CD58 and CD40, and fully activate cytotoxic T-lymphocytes (CTLs) immune response against cancer cells [6]. Therefore, cancer immunotherapy involving of DCs acts as a promising method. The DC-based strategies not only prevent cancer recurrence, but also reduce its progression and metastasis [7-10]. Meanwhile, cytokines secreted from DCs play a vital role in antitumor response [11]. Moreover, tumor-infiltrating DCs (TIDC) are associated with predictable clinical outcome, prolonged survival and reduced tumor recurrence [12-14]. On the other hand, dysfunction of DCs can lead to ineffective $\mathrm{T}$ cell activation. In this case, $\mathrm{T}$ cell cannot recognize cancer cells and initiate defense response. Previous studies demonstrated that several malignant tumors could inhibit phenotype and function of DCs [15-17].

AG490 is a Jak2/STAT3 inhibitor, which can activate DCs by inducing cancer cell death [18], and suppress progression of bladder transitional cell carcinomas [19, 20]. Activated STAT3 signaling is related to bladder transitional cell carcinomas growth 
and survival [21]. Therefore, interrupting STAT3 signaling can suppress cancer drug resistance, growth and metastasis [22-24].

Until now, the effect of bladder cancer cell (BCC) on human DCs remains unclear. In our study, DCs were co-cultured with BCC pumc-91 and adriamycin-resistant pumc-91/ADM. The phenotype and function of DCs were evaluated thereafter. Moreover, we also investigated the immunosuppressive effect of BCC on DCs might involve of Jak2/STAT3 pathway.

\section{RESULTS}

\section{Inhibition maturation of human DCs by co-culture with $\mathrm{BCC}$}

We first evaluated the effect of $\mathrm{BCC}$ on $\mathrm{DC}$ maturation. Compared with control DCs, pumc-91-exposed DCs displayed reduced expression of CD11c, HLA-DR and CD86. Besides that, the expression of HLA-ABC further decreased in DCs co-cultured with pumc-91/ ADM. However, there were no differences between control DCs and DCs co-cultured with SV-HUC-1, a human uroepithelial cell line (Figure 1A). In supernatant experiment, compared with control DCs and SV-HUC-1 cs-exposed DC, the pumc-91cs-exposed DCs expressed lower levels of HLA-DR, CD11c and CD86, while the expression of HLA-ABC further decreased in pumc-91/ ADM cs-exposed DCs. (Figure 1B). These data indicate that not only bladder cancer cell, but also the soluble factors from BCC inhibited the maturation of human DCs, and there might be different mechanisms by which adriamycin-resistant BCC inhibits DC maturation.

\section{BCC induced apoptosis of human DCs}

As cancer cells can escape immune surveillance by inducing DC apoptosis [25]. We next tested the effect of $\mathrm{BCC}$ on DC apoptosis. The apoptosis rate of DCs was assessed by the percentage of FITC-Annexin V positive cells, while the dead cells identified with propidium iodide. As shown in Figure 2, DCs co-cultured with BCC induced higher proportion of apoptotic cells in comparison to control DCs, and BCC promoted DC apoptosis.

\section{The effect of BCC on the production of DCs-derived chemokines}

In addition to HLA molecules and co-stimulatory molecules, chemokines secreted from DCs also play an important role in antitumor immune response. Supernatants of control DCs, SV-HUC-1 and BCCexposed DCs were analyzed for production of IP-10, MCP-1, MIG, RANTES and IL-8. Compared with control DCs and SV-HUC-1-exposed DCs, BCC-exposed DCs secreted lower levels of IL-8 and RANTES. Interestingly, compared with control DCs and SV-HUC-1-exposed DCs, DCs co-cultured with pumc-91 produced more IP-10, while DCs co-cultured with pumc-91/ADM secreted more MIG (Figure 3). These data demonstrate that BCC could affect the production of DCs-derived chemokines, and there might be different mechanisms by which adriamy cin-resistant BCC inhibits DC function.

\section{Inhibition of $T$ cell proliferation by BCC-exposed DCs}

We next examined the immunostimulatory effect of DCs co-cultured with BCC. Control DCs, SV-HUC-1 and $\mathrm{BCC}$-exposed DCs were used to stimulate allogeneic $\mathrm{T}$ cells. As shown in Figure 4, compared with control DCs and SV-HUC-1-exposed DCs, BCC co-cultured DCs showed impaired allogeneic $\mathrm{T}$ cell proliferation. These results demonstrated that $\mathrm{BCC}$ suppressed the capacity of DCs, leading to ineffective $\mathrm{T}$ cell activation.

\section{The effect of BCC-exposed DCs on T cell-derived cytokines}

To further examine the immunostimulatory effect of BCC-exposed DCs, we measured the levels of T cellderived cytokines in above $\mathrm{T}$ cell-DC co-culture system by using the CBA human Th1/Th2/Th17 cytokine kit. We found that $\mathrm{BCC}$ exposed-DCs induced $\mathrm{T}$ cells secreting less cytokines than control DCs and SV-HUC-1-exposed DCs, including IL-2, IL-4, IL-6, IL-10, TNF- $\alpha$, IFN- $\gamma$, and IL-17A (Figure 5). These data indicate that BCCexposed DCs inhibited $\mathrm{T}$ cell to produce Th1, Th2 and Th17 cytokines.

\section{Immunosuppressive effect of BCC on DCs may through Jak2/STAT3 pathway}

To explore the pathway of $\mathrm{BCC}$ inhibiting DC function. We prior treated pumc-91 or pumc-91/ADM with AG490, a Jak2/STAT3 inhibitor, and then co-cultured BCC with DCs. As shown in Figure 6A, compared with pumc-91-exposed DCs, the expression of CD11c, CD86 and HLA-DR were increased in DCs co-cultured with pumc-91/AG490; while in the pumc-91/ADM group, compared with pumc-91/ADM-exposed DCs, pumc91/ADM/AG490-exposed DCs displayed increased expression of CD86 and HLA-DR (Figure 6B).

\section{DISCUSSION}

Mature DCs activate tumor antigen-specific T cell to induce antitumor immune. However, immature DCs may lead to deficient activation of specific cytotoxic T cells, thus suppress antitumor immune response and promote cancer growth and progression $[8,10]$. Recent studies reported that some cancers could induce immaturity and 
A

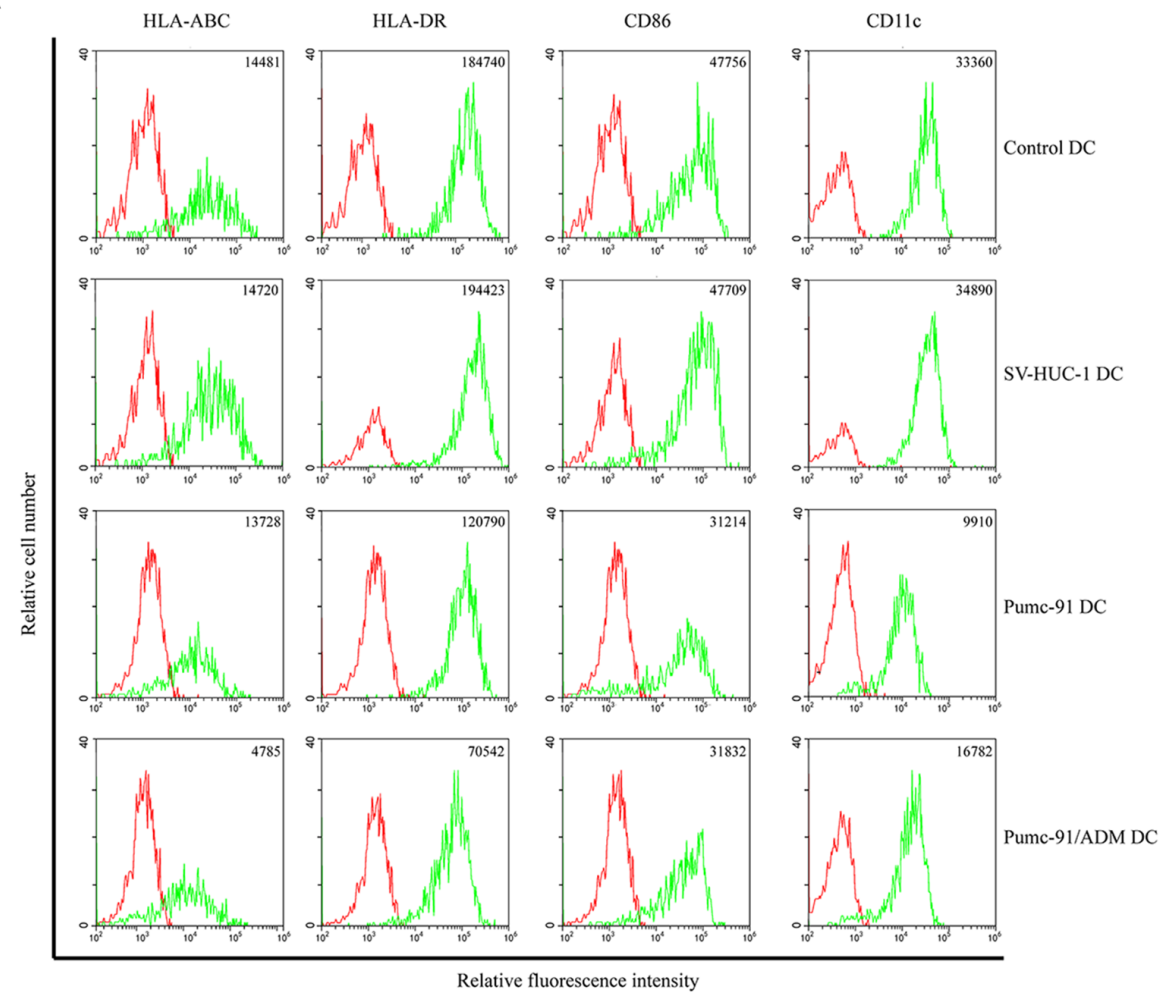

B

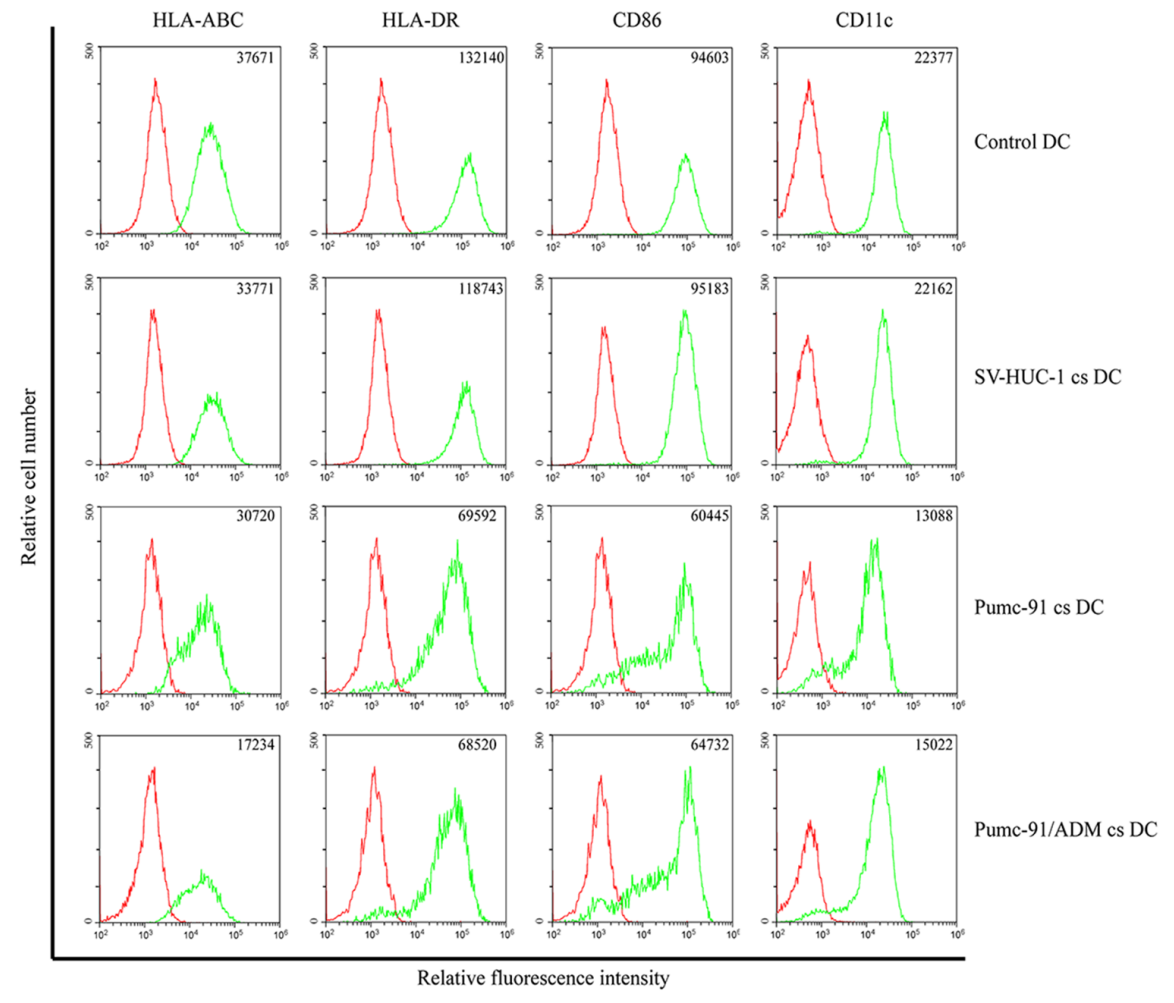

Figure 1: Effect of BCC on DCs phenotype. (A) DCs were treated with SV-HUC-1 or BCC for 5 days and then induced with LPS for $24 \mathrm{~h}$. The expressions of cell surface markers were analyzed by FACS. The mean fluorescence intensity (MFI) are present in the top right of each panel. Data shown are a representative experiment of three. (B) DCs cultured with supernatants of SV-HUC-1 or BCC. The cells were harvested and surface markers were analyzed by FACS. The mean fluorescence intensity (MFI) are present in the top right of each panel. Data shown are a representative experiment of three. 

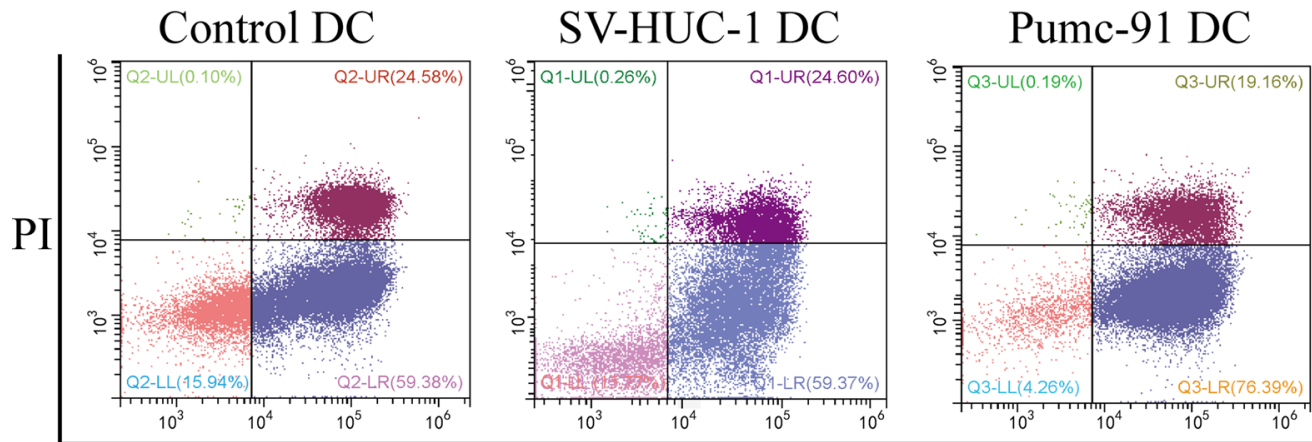

Pumc-91/ADM DC

Annexin V FITC

Figure 2: Increased apoptosis of DCs co-cultured with BCC. Control DCs, SV-HUC-1 or BCC-exposed DCs were stained with FITC-Annexin-V and propidium iodide (PI). Apoptotic cells were increased in BCC-exposed DCs. Data shown are a representative experiment of three.
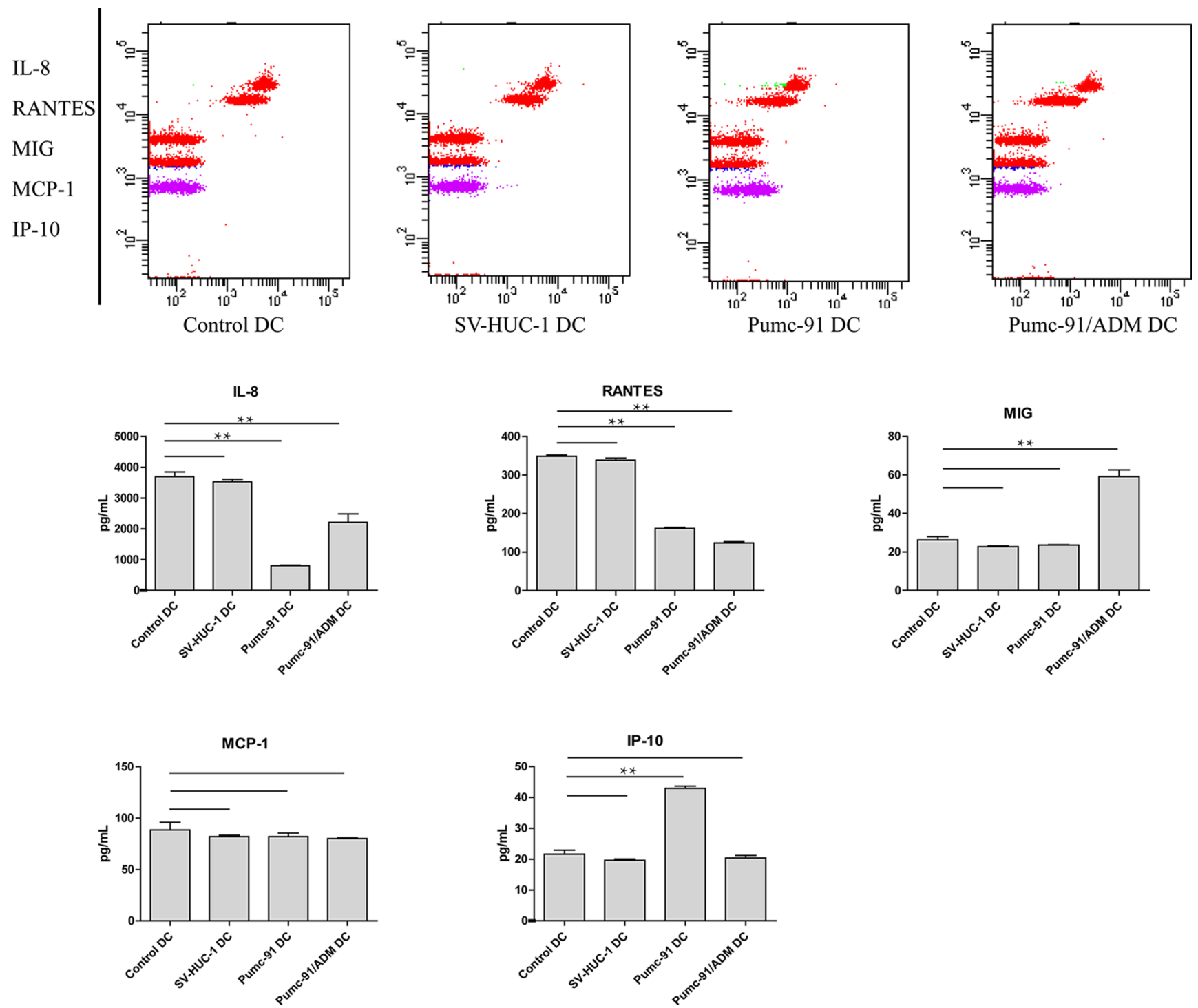

Figure 3: Production of chemokines IL-8, RANTES, MIG, MCP-1 and IP-10 by BCC-exposed DCs. Control DCs, SV-HUC-1 or BCC-exposed DCs was induced by LPS. Cytometric Bead Array (CBA) was used to analyze concentrations of chemokines in cell-free supernatants. Values shown are mean \pm standard deviation (SD) from triplicate representative experiments. $* * P<0.01$. 


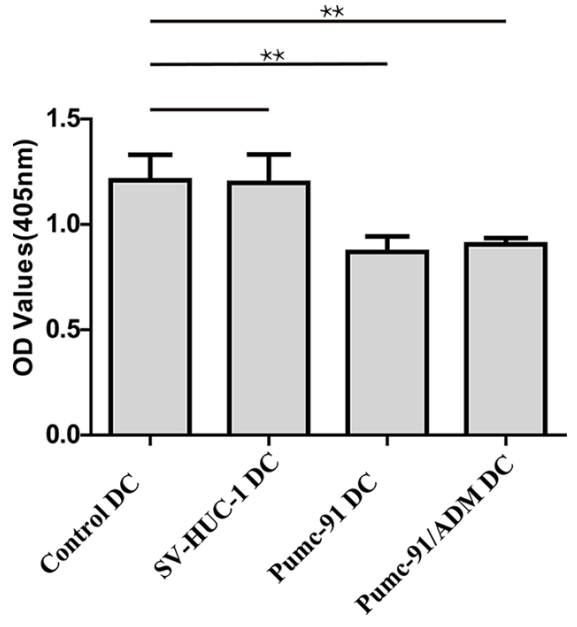

Figure 4: Reduced allogeneic T cells proliferation stimulated by DCs co-cultured with BCC. Control DCs, SV-HUC-1 or BCC-exposed DCs were treated with LPS. Then purified and irradiated (30Gy) DCs were co-cultured with allogeneic T cells for 5 days. $\mathrm{T}$ cell proliferation was measured at $450 \mathrm{~nm}$ by CCK- 8 . Values shown are mean \pm standard deviation (SD) from triplicate representative experiments. $* * P<0.01$.
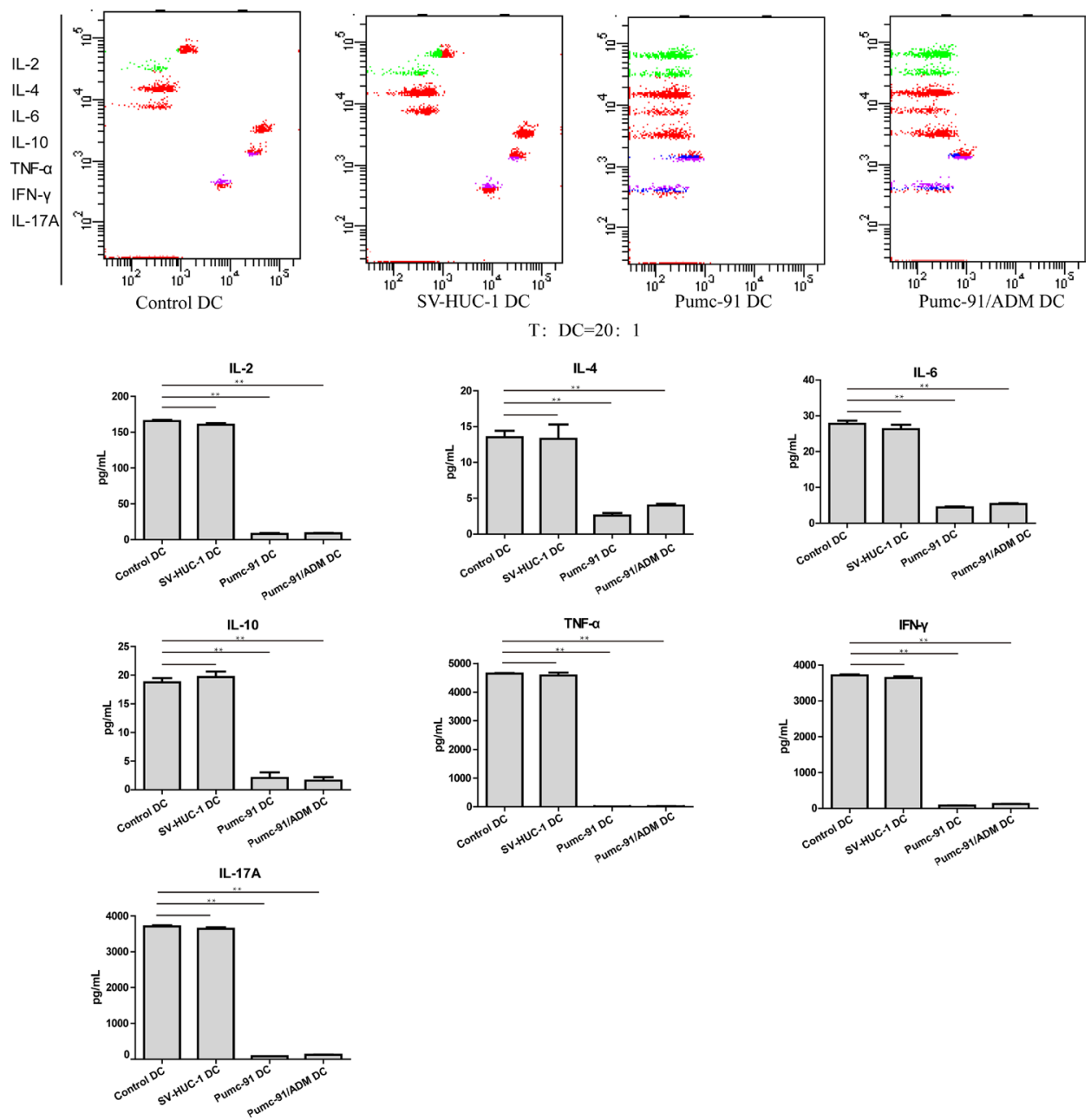

Figure 5: Reduced cytokine production of by allogeneic T cells induced by BCC-exposed DCs. Control DCs, SV-HUC-1 or BCC-exposed DCs was treated with LPS. Purified and irradiated (30 Gy) DCs were co-cultured with allogeneic T cells for 5 days. The concentrations of cytokines in cell-free supernatants were measured by CBA human Th1/Th2/Th17 Cytokine Kit. Values shown are mean \pm standard deviation (SD) from triplicate representative experiments. $* * P<0.01$. 


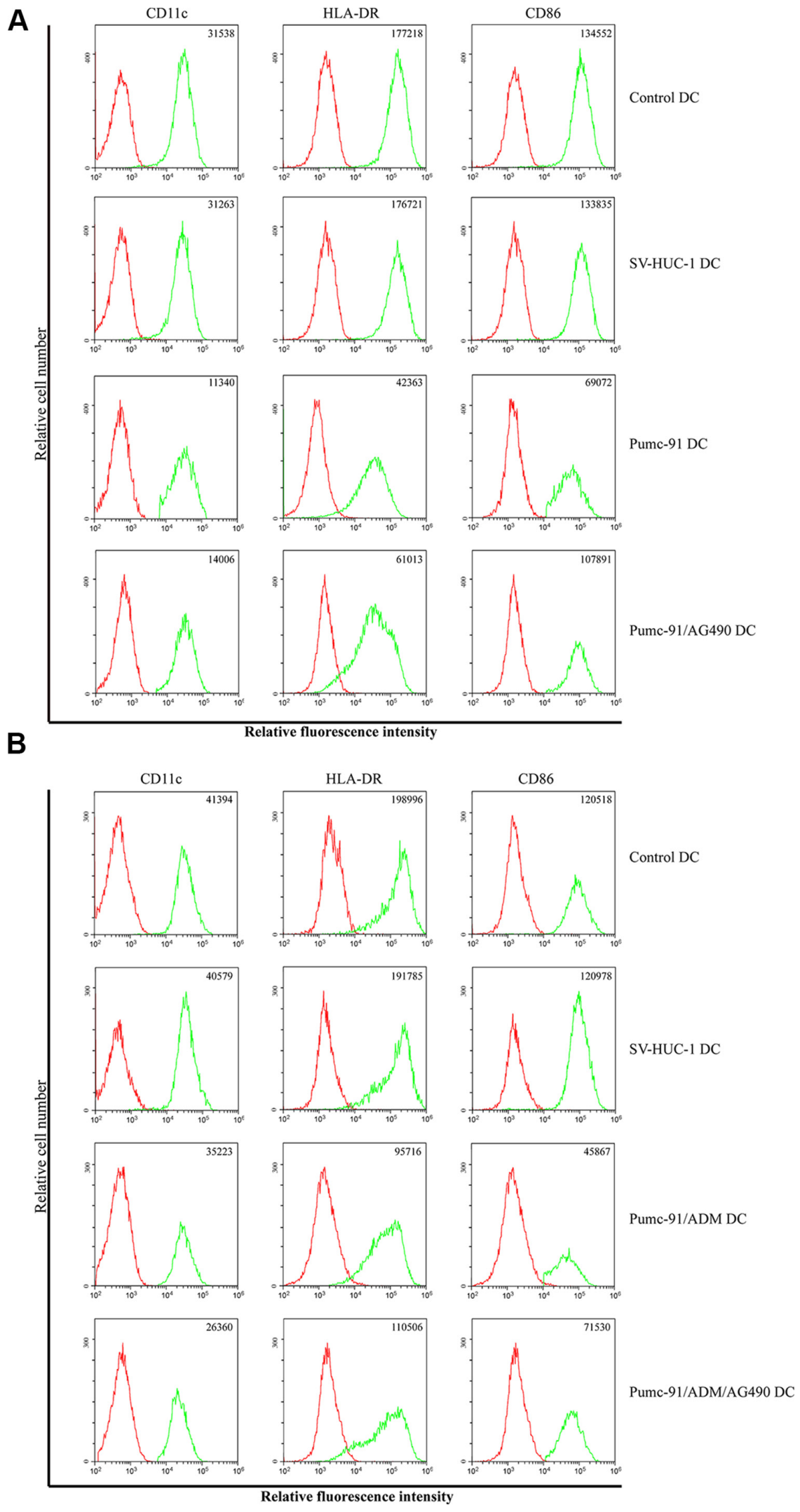

Figure 6: Effects of AG490 on BCC-exposed DCs phenotype. Pumc-91 (A) or Pumc-91/ADM (B) prior treated with AG490 and co-cultured with DCs for $24 \mathrm{~h}$, the expressions of DCs surface markers were analyzed by FACS. The mean fluorescence intensity (MFI) are present in the top right of each panel. Data shown are a representative experiment of three. 
dysfunction of DCs. Uveal melanoma cell co-cultured DCs exhibited decreased expression of CD1a and CD83 and impaired $\mathrm{T}$ cell activation [16]. In our study, we identified the impairments in the phenotype of DCs co-cultured with BCC. We found that DCs exposed to BCC exhibited an immature phenotype. Compared with control DCs, DCs co-cultured with pumc-91 showed lower expression of CD11c, HLA-DR and CD86, while DCs co-cultured with pumc-91/ADM further displayed declined expression of HLA-ABC. In addition to the interactions between bladder cancer cells and DCs, DCs might be affected by soluble factors secreted from bladder cancer cells. DCs cultured with supernatants of pumc-91 and pumc-91/ ADM showed similar impairment function. These data suggest that pumc-91 and pumc-91/ADM could suppress DC maturation. Interestingly, immunosuppressive effect of pumc-91 or pumc-91/ADM on maturation of DCs seems to be different. Of note, inhibition of DC maturation may be the characteristics of some tumors, while others not. Retinoblastoma cell could upregulate CD80 and CD86 on DCs, and stimulated allogeneic T cells proliferation [26].

A series of researches have been demonstrated that DCs undergo apoptosis after treated by cancer cells $[25,27]$. Indeed, to evade antitumor immunity, cancer can induce DCs to undergo apoptosis, and thus lead to dysfunction of DCs [27]. BCC may utilize this mechanism to promote DCs apoptosis so that they could escape from immune surveillance. This may in part explain why T cells fail to become fully activated in our experiment.

As an effective inhibitor for Jak2/STAT3 pathway, AG490 reversed pancreatic cancer cell-induced inhibition of DC differentiation [28]. In our group, BCC decreased expression of costimulatory molecules on DCs, whereas BCC prior treated with AG490 could reverse the reduction of some molecules. This suggests that Jak2/STAT3 pathway, at least partly played a critical role in inducing immunosuppression effect of BCC. Furthermore, immunogenic apoptosis have a great effect on tumor microenvironment [29-31]. Compared to the 'classical' apoptosis, immunogenic apoptosis can induce DCs-based antitumor response. Bortezomib-killed tumor cells could be induced apoptosis, then activated DCs and enhanced antitumor T-cell response [32]. Prior treated with AG490 could cause apoptosis of lymphoma cells and promote DC maturation [18]. Accordingly, based on these findings, we speculate there may be same mechanisms by which AG490 effects on BCC and DCs.

CXCL8/IL-8 has multiple biological functions. On one hand, IL-8 led to aggressive growth and metastasis of human malignant melanoma [33]. On the other hand, IL-8 could inhibit growth and proliferation of some cancers [34, 35]. CCL5/RANTES is proinflammatory C-C chemokine. Several researches have demonstrated RANTES could delay or inhibit tumor growth and enhance the immunological antitumor effect $[36,37]$. In our group, compared with control DCs, DCs co-cultured with BCC secreted less IL-8 and RANTES. Interestingly, compared with control DCs, pumc-91-exposed DCs secreted more IP-10, while pumc-91/ADM-exposed DCs produced more MIG. CXCL10/IP-10 is one of the Th1-attracting cytokines, and IFN- $\gamma$ enhances expression of IP-10 during DCs maturation [38, 39]. IP-10 promoted invasion-related properties in human colorectal cancer cells [40]. Pumc-91 might utilize the properties to enhance its invasiveness. CXCL9/MIG induces tumor necrosis and exerts an important effect on antitumor response in Burkitt's lymphoma [41]. Conversely, increased MIG could facilitate melanoma cell metastasis through CXCR3 [42]. Accordingly, immunologic function of MIG may depend on the characteristics of cancer. MIG secreted from pumc91/ADM-exposed DCs might trigger cancer migration and invasion. These findings implied that the immunosuppressive effect of pumc-91 or pumc-91/ADM on DCs might have different mechanisms. Moreover, our study also suggested that it is necessary to supply different immunotherapy methods for drug-resistant bladder cancer patients.

In our study, the expression of CD86 decreased in BCC-exposed DCs. Interaction of CD86 and CD80 with CD28 led to the activation and proliferation of $\mathrm{T}$ cells $[43,44]$. Keep in line with these findings, we found that there was impaired allogeneic $\mathrm{T}$ cells proliferation when $\mathrm{T}$ cells were co-cultured with BCC-exposed DCs. Our study suggested that $\mathrm{BCC}$ could induce DC dysfunction, failing to induce $\mathrm{T}$ cell response. Moreover, BCC-exposed DCs underwent rapid apoptosis which may reduce the time window during which they interact with T cells [45]. In addition, we observed that $\mathrm{T}$ cells secreted less cytokines when co-cultured with BCC-exposed DCs. The data suggest that BCC restrain DCs' ability to activate T cells.

In summary, our study suggests BCC may inhibit maturation and function of DCs involving of Jak2/STAT3 pathway, and there may be different mechanisms by which adriamycin-resistant $\mathrm{BCC}$ restrains DC function in antitumor immune response.

\section{MATERIALS AND METHODS}

\section{Cell culture}

The SV-40 immortalized human uroepithelial cell line (SV-HUC-1) was obtained from Chinese Academy of Sciences Cell Bank (CASCB, China). SV-HUC-1 cells were cultured in Ham's F-12 (Sigma, USA) medium supplemented with $10 \%$ heat-inactivated fetal bovine serum (FBS) (Ausbian, Australia) at $37^{\circ} \mathrm{C}$ and $5 \%$ $\mathrm{CO}_{2}$. The human bladder cancer pumc-91 cell line was provided by Peking Union Medical College Hospital. The drug resistant cell line was pumc-91/ADM, which established by increasing the dosage of adriamycin gradually, and the final concentration of adriamycin was $1.0 \mu \mathrm{g} / \mathrm{ml}[46,47]$. SV-HUC-1 cell supernatant (SV-HUC-1cs), pumc-91 cell supernatant (Pumc-91cs) or 
pumc-91/ADM cell supernatant (Pumc-91/ADM cs) were prepared by seeding $10-\mathrm{cm}$ dish (Corning, NY, USA) with $1 \times 10^{7} \mathrm{SV}-\mathrm{HUC}-1$ cell, pumc-91 cell or pumc91/ADM cell in $10 \mathrm{ml}$ of RPMI 1640 medium (Gibco, USA) for 24 hours and centrifuged to remove cells. Peripheral blood samples were from healthy individuals and peripheral blood mononuclear cells (PBMCs) were isolated immediate by Ficoll-Hypaque density gradient centrifugation. Subsequently, PBMCs were washed triple with AIM-V medium (Gibco, USA), suspended in AIM-V medium at $5 \times 10^{6}$ cells $/ \mathrm{ml}$, and seeded in a 6-well plate (greiner bio-one, Germany) at $2 \mathrm{ml}$ per well. The plate was incubated at $37^{\circ} \mathrm{C}$ for $2 \mathrm{~h}$, and the nonadherent cells were discarded. The adherent cells were cultured for 6 days in AIM-V medium supplemented with $10 \%$ heat-inactivated FBS, $50 \mathrm{ng} / \mathrm{ml} \mathrm{rhGM}-\mathrm{CSF}$ and $50 \mathrm{ng} / \mathrm{ml} \mathrm{rhIL-4}$ (Peprotech, USA) to obtain immature DCs (iDCs). Half-volume medium exchange was performed every 3 days with AIM-V medium containing fresh cytokines.

\section{BCC treatment with AG490}

AG490 (Sigma, USA) was dissolved in dimethylsulfoxide (DMSO) (Sigma, USA) and diluted to final concentrations of $200 \mu \mathrm{M}$. Pumc-91 or pumc-91/ ADM was treated with AG490 for $24 \mathrm{~h}$. The AG490treated pumc-91 (pumc-91/AG490) or pumc-91/ADM (pumc-91/ADM/AG490) were washed twice and then co-cultured with DCs for $24 \mathrm{~h}$. Background control group was $1.2 \%$ DMSO treated with pumc-91 or pumc91/ADM.

\section{Co-cultured of dendritic cells with BCC}

On day 5 of DCs culture, $1 \times 10^{6}$ pumc-91 or pumc91/ADM or SV-HUC-1 were added to each well of DCs. In a separate experiment, the cell-free supernatant of BCC was added to test DCs, while the same volume of AIM-V medium or SV-HUC-1cs was added to control DCs. On day 6 , maturation of DCs was induced by $1 \mu \mathrm{g} / \mathrm{ml}$ of LPS (Sigma, USA) for $24 \mathrm{~h}$. In most experiments, control DCs, BCC co-cultured DCs were washed and then purified with microbeads on QuadroMACS Starting kit (LD) using a Blood Dendritic Isolation kit (Miltenyi Biotech, Germany). The cell suspension was then passed through MiniMACS Starting kit (Miltenyi Biotech, Germany). According to the manufacturer's instruction, cells were labeled with the Non-DC Depletion Cocktail, comprising CD1c (BDCA-1), CD14 and CD19 microbeads for negative selected. Then cells were labeled with DC Enrichment Cocktail, comprising CD304 (BDCA-4/Neuropilin-1), CD141 (BDCA-3) and Biotin microbeads for positive selected. The DCs samples contain more than $95 \% \mathrm{CD}^{\mathrm{c}} \mathrm{c}^{+} \mathrm{DCs}$ in these experiments.

\section{Flow cytometry analysis of DCs phenotype}

Phenotype of DCs co-cultured with BCC was analyzed by FACS. The following test antibodies conjugated with either allophycocyanin (APC) or phycoerythrin (PE) was used: CD11c, CD86, HLA-ABC, HLA-DR and isotype control mAbs (eBioscience, San Diego, CA). The phenotype of DCs was tested by staining with antibodies for 30 minutes at $4^{\circ} \mathrm{C}$, and then washed twice in phosphate buffered saline (PBS). Samples were analyzed by a flow cytometer (CytoFLEX, Beckman coulter) and data were processed using accompanying software (CytExpert, Beckman coulter).

\section{Apoptosis assay}

To examine apoptosis, DCs were washed with icecold PBS, stained by FITC Annexin V Apoptosis Detection kit I (BD Pharmingen) according to the manufacturer's instruction. Samples were analyzed by the flow cytometer (CytoFLEX, Beckman coulter) and data were processed using the accompanying software (CytExpert, Beckman coulter).

\section{DC-derived chemokine assays}

Purified DCs were cultured in 6 well plates at a density of $1.8 \times 10^{5}$ cells/well in $2 \mathrm{ml}$ of AIM-V medium. Cell free supernatant was harvested after induced maturation by using LPS. The level of IP-10, MCP-1, MIG, RANTES and IL-8 were measured by Cytometric Bead Array (CBA) Human Chemokine kit (BD bioscience) according to the manufacturer's instruction. Samples were analyzed using a flow cytometer (FACS Canto, BD Bioscience) and data were processed by accompanying software (FCAP Array; BD Bioscience).

\section{T cell proliferation assay}

Heparinized peripheral blood samples were from healthy individuals and PBMCs were isolated immediate by Ficoll-Hypaque density gradient centrifugation. Pan T cell Isolation kit (Miltenyi Biotech) was used to separate $\mathrm{CD}^{+} \mathrm{T}$ cells according to the protocols recommended by manufacturer. X-irradiator (Gammacell 40 Exactor; MDS Nordion International, Inc., Ottawa, Ontario, CA) irradiated the purified DCs for 30 minutes at $30 \mathrm{G}$. Purified $\mathrm{CD}^{+} \mathrm{T}$ cells seeded into a round-bottom 96 well plate (greiner bio-one, Germany) at $2.0 \times 10^{5}$ per well. Then purified $\mathrm{CD}^{+} \mathrm{T}$ cells were co-cultured with purified, stimulated and irradiated DCs at the ratio of 20:1. Background control group was $\mathrm{T}$ cells alone. The culture system was incubated for 5 days at $37^{\circ} \mathrm{C}$ in $5 \% \mathrm{CO} 2$ in air. The proliferations of $\mathrm{T}$ cells were analyzed using Cell Counting Kit-8 (CCK-8) (Dojindo, Kumamoto, Japan) during the last $4 \mathrm{~h}$ of incubation. 


\section{T cell-derived cytokine assays}

Cell free supernatant was harvested from T cell-DC co-culture system after $72 \mathrm{~h}$. The level of IL-2, IL-4, IL-6, IL-10, IL-17, TNF- $\alpha$ and IFN- $\gamma$ were measured by Cytometric Bead Array (CBA) Human Th1/Th2/ Th17 Cytokine kit (BD bioscience) according to the manufacturer recommendation. Samples were analyzed using a flow cytometer (FACS Canto, BD Bioscience) and data were processed by the accompanying software (FCAP Array; BD Bioscience).

\section{Statistical analysis}

All the experiments were repeated three times. Oneway ANOVA was used to estimate all analyses. $P<0.05$ was accepted as statistical significance.

\section{ACKNOWLEDGMENTS AND FUNDING}

This work was supported by Beijing key Laboratory of Urinary Cellular Molecular Diagnostics (Z151100001615060) and National High Technology Research and Development Program of China (2014AA020901), and the National Nature Science Foundation of China (No.31400754).

\section{CONFLICTS OF INTEREST}

The authors declare no conflicts of interest.

\section{REFERENCES}

1. Torre LA, Bray F, Siegel RL, Ferlay J, Lortet-Tieulent J, Jemal A. Global cancer statistics, 2012. CA Cancer J Clin. 2015; 65:87-108.

2. Sylvester RJ, van der Meijden AP, Oosterlinck W, Witjes JA, Bouffioux C, Denis L, Newling DW, Kurth K. Predicting recurrence and progression in individual patients with stage Ta T1 bladder cancer using EORTC risk tables: a combined analysis of 2596 patients from seven EORTC trials. European urology. 2006; 49:466-465; discussion 475-467.

3. Jin X, Yun SJ, Jeong P, Kim IY, Kim WJ, Park S. Diagnosis of bladder cancer and prediction of survival by urinary metabolomics. Oncotarget. 2014; 5:1635-1645. doi: 10.18632/oncotarget.1744.

4. Tada Y, Wada M, Migita T, Nagayama J, Hinoshita E, Mochida Y, Maehara Y, Tsuneyoshi M, Kuwano M, Naito S. Increased expression of multidrug resistance-associated proteins in bladder cancer during clinical course and drug resistance to doxorubicin. International journal of cancer. 2002; 98:630-635.

5. Li Y, Deng H, Lv L, Zhang C, Qian L, Xiao J, Zhao W, Liu Q, Zhang D, Wang Y, Yan J, Zhang H, He Y, et al. The miR-193a-3p-regulated ING5 gene activates the DNA damage response pathway and inhibits multi-chemoresistance in bladder cancer. Oncotarget. 2015; 6:10195-10206. doi: 10.18632/oncotarget.3555.

6. Banchereau J, Palucka AK. Dendritic cells as therapeutic vaccines against cancer. Nature reviews Immunology. 2005; 5:296-306.

7. Ho PL, Williams SB, Kamat AM. Immune therapies in nonmuscle invasive bladder cancer. Current treatment options in oncology. 2015; 16:5.

8. Thompson DB, Siref LE, Feloney MP, Hauke RJ, Agrawal DK. Immunological basis in the pathogenesis and treatment of bladder cancer. Expert review of clinical immunology. 2015; 11:265-279.

9. Kantoff PW, Higano CS, Shore ND, Berger ER, Small EJ, Penson DF, Redfern CH, Ferrari AC, Dreicer R, Sims RB, Xu Y, Frohlich MW, Schellhammer PF, et al. Sipuleucel-T immunotherapy for castration-resistant prostate cancer. The New England journal of medicine. 2010; 363:411-422.

10. Palucka K, Banchereau J. Cancer immunotherapy via dendritic cells. Nature reviews Cancer. 2012; 12:265-277.

11. Nishioka Y, Hirao M, Robbins PD, Lotze MT, Tahara H. Induction of systemic and therapeutic antitumor immunity using intratumoral injection of dendritic cells genetically modified to express interleukin 12. Cancer research. 1999; 59:4035-4041.

12. Tran Janco JM, Lamichhane P, Karyampudi L, Knutson KL. Tumor-infiltrating dendritic cells in cancer pathogenesis. Journal of immunology (Baltimore, Md : 1950). 2015; 194:2985-2991.

13. Ladanyi A, Kiss J, Somlai B, Gilde K, Fejos Z, Mohos A, Gaudi I, Timar J. Density of DC-LAMP $(+)$ mature dendritic cells in combination with activated $\mathrm{T}$ lymphocytes infiltrating primary cutaneous melanoma is a strong independent prognostic factor. Cancer immunology, immunotherapy. 2007; 56:1459-1469.

14. Ma Y, Shurin GV, Peiyuan Z, Shurin MR. Dendritic cells in the cancer microenvironment. Journal of Cancer. 2013; 4:36-44.

15. Katsenelson NS, Shurin GV, Bykovskaia SN, Shogan J, Shurin MR. Human small cell lung carcinoma and carcinoid tumor regulate dendritic cell maturation and function. Modern pathology. 2001; 14:40-45.

16. Ma J, Usui Y, Takeuchi M, Okunuki Y, Kezuka T, Zhang L, Mizota A, Goto H. Human uveal melanoma cells inhibit the immunostimulatory function of dendritic cells. Experimental eye research. 2010; 91:491-499.

17. Teng L, Chen Y, Ding D, Dai H, Liu G, Li C. Immunosuppressive effect of renal cell carcinoma on phenotype and function of dendritic cells. International urology and nephrology. 2014; 46:915-920.

18. Cirone M, Di Renzo L, Lotti LV, Conte V, Trivedi P, Santarelli R, Gonnella R, Frati L, Faggioni A. Primary effusion lymphoma cell death induced by bortezomib and AG 490 activates dendritic cells through CD91. PloS one. 2012; 7:e31732. 
19. Joung YH, Na YM, Yoo YB, Darvin P, Sp N, Kang DY, Kim SY, Kim HS, Choi YH, Lee HK, Park KD, Cho BW, Kim HS, et al. Combination of AG490, a Jak2 inhibitor, and methylsulfonylmethane synergistically suppresses bladder tumor growth via the Jak2/STAT3 pathway. International journal of oncology. 2014; 44:883-895.

20. Sun Y, Cheng MK, Griffiths TR, Mellon JK, Kai B, Kriajevska M, Manson MM. Inhibition of STAT signalling in bladder cancer by diindolylmethane: relevance to cell adhesion, migration and proliferation. Current cancer drug targets. 2013; 13:57-68.

21. Chen CL, Cen L, Kohout J, Hutzen B, Chan C, Hsieh FC, Loy A, Huang V, Cheng G, Lin J. Signal transducer and activator of transcription 3 activation is associated with bladder cancer cell growth and survival. Molecular cancer. 2008; 7:78.

22. Song H, Wang R, Wang S, Lin J. A low-molecular-weight compound discovered through virtual database screening inhibits Stat3 function in breast cancer cells. Proceedings of the National Academy of Sciences of the United States of America. 2005; 102:4700-4705.

23. Jing N, Tweardy DJ. Targeting Stat3 in cancer therapy. Anticancer drugs. 2005; 16:601-607.

24. Chai EZ, Shanmugam MK, Arfuso F, Dharmarajan A, Wang C, Kumar AP, Samy RP, Lim LH, Wang L, Goh BC, Ahn KS, Hui KM, Sethi G. Targeting transcription factor STAT3 for cancer prevention and therapy. Pharmacology \& therapeutics. 2016.

25. Kiertscher SM, Luo J, Dubinett SM, Roth MD. Tumors promote altered maturation and early apoptosis of monocyte-derived dendritic cells. Journal of immunology (Baltimore, Md : 1950). 2000; 164:1269-1276.

26. Ma J, Han H, Ma L, Liu C, Xue X, Ma P, Li X, Tao H. The immunostimulatory effects of retinoblastoma cell supernatant on dendritic cells. Protein \& cell. 2014; 5:307-316.

27. Pinzon-Charry A, Maxwell T, McGuckin MA, Schmidt C, Furnival C, Lopez JA. Spontaneous apoptosis of blood dendritic cells in patients with breast cancer. Breast cancer research. 2006; 8:R5.

28. Bharadwaj U, Li M, Zhang R, Chen C, Yao Q. Elevated interleukin-6 and G-CSF in human pancreatic cancer cell conditioned medium suppress dendritic cell differentiation and activation. Cancer research. 2007; 67:5479-5488.

29. Kroemer G, Galluzzi L, Kepp O, Zitvogel L. Immunogenic cell death in cancer therapy. Annual review of immunology. 2013; 31:51-72.

30. Schiavoni G, Sistigu A, Valentini M, Mattei F, Sestili P, Spadaro F, Sanchez M, Lorenzi S, D’Urso MT, Belardelli F, Gabriele L, Proietti E, Bracci L. Cyclophosphamide synergizes with type I interferons through systemic dendritic cell reactivation and induction of immunogenic tumor apoptosis. Cancer research. 2011; 71:768-778.

31. Apetoh L, Mignot G, Panaretakis T, Kroemer G, Zitvogel L. Immunogenicity of anthracyclines: moving towards more personalized medicine. Trends in molecular medicine. 2008; 14:141-151.

32. Spisek R, Charalambous A, Mazumder A, Vesole DH, Jagannath S, Dhodapkar MV. Bortezomib enhances dendritic cell (DC)-mediated induction of immunity to human myeloma via exposure of cell surface heat shock protein 90 on dying tumor cells: therapeutic implications. Blood. 2007; 109:4839-4845.

33. Varney ML, Johansson SL, Singh RK. Distinct expression of CXCL8 and its receptors CXCR1 and CXCR2 and their association with vessel density and aggressiveness in malignant melanoma. American journal of clinical pathology. 2006; 125:209-216.

34. Lee LF, Hellendall RP, Wang Y, Haskill JS, Mukaida N, Matsushima K, Ting JP. IL-8 reduced tumorigenicity of human ovarian cancer in vivo due to neutrophil infiltration. Journal of immunology (Baltimore, Md : 1950). 2000; 164:2769-2775.

35. Wang J, Huang M, Lee P, Komanduri K, Sharma S, Chen G, Dubinett SM. Interleukin-8 inhibits non-small cell lung cancer proliferation: a possible role for regulation of tumor growth by autocrine and paracrine pathways. Journal of interferon \& cytokine research. 1996; 16:53-60.

36. Mule JJ, Custer M, Averbook B, Yang JC, Weber JS, Goeddel DV, Rosenberg SA, Schall TJ. RANTES secretion by gene-modified tumor cells results in loss of tumorigenicity in vivo: role of immune cell subpopulations. Human gene therapy. 1996; 7:1545-1553.

37. Inoue H, Iga M, Xin M, Asahi S, Nakamura T, Kurita R, Nakayama M, Nakazaki Y, Takayama K, Nakanishi Y, Tani K. TARC, RANTES enhance antitumor immunity induced by the GM-CSF-transduced tumor vaccine in a mouse tumor model. Cancer immunology, immunotherapy. 2008; 57:1399-1411.

38. Vandercappellen J, Van Damme J, Struyf S. The role of CXC chemokines and their receptors in cancer. Cancer letters. 2008; 267:226-244.

39. Padovan E, Spagnoli GC, Ferrantini M, Heberer M. IFN-alpha2a induces IP-10/CXCL10 and MIG/CXCL9 production in monocyte-derived dendritic cells and enhances their capacity to attract and stimulate CD8+ effector T cells. Journal of leukocyte biology. 2002; 71:669-676.

40. Zipin-Roitman A, Meshel T, Sagi-Assif O, Shalmon B, Avivi C, Pfeffer RM, Witz IP, Ben-Baruch A. CXCL10 promotes invasion-related properties in human colorectal carcinoma cells. Cancer research. 2007; 67:3396-3405.

41. Sgadari C, Farber JM, Angiolillo AL, Liao F, TeruyaFeldstein J, Burd PR, Yao L, Gupta G, Kanegane C, Tosato G. Mig, the monokine induced by interferongamma, promotes tumor necrosis in vivo. Blood. 1997; 89:2635-2643.

42. Kawada K, Sonoshita M, Sakashita H, Takabayashi A, Yamaoka Y, Manabe T, Inaba K, Minato N, Oshima M, 
Taketo MM. Pivotal role of CXCR3 in melanoma cell metastasis to lymph nodes. Cancer research. 2004; 64:4010-4017.

43. Collins M, Ling V, Carreno BM. The B7 family of immuneregulatory ligands. Genome biology. 2005; 6:223.

44. Orabona C, Grohmann U, Belladonna ML, Fallarino F, Vacca C, Bianchi R, Bozza S, Volpi C, Salomon BL, Fioretti MC, Romani L, Puccetti P. CD28 induces immunostimulatory signals in dendritic cells via CD80 and CD86. Nature immunology. 2004; 5:1134-1142.

45. Pinzon-Charry A, Maxwell T, Lopez JA. Dendritic cell dysfunction in cancer: a mechanism for immunosuppression. Immunology and cell biology. 2005; 83:451-461.
46. Yu S, Meng Q, Hu H, Zhang M. Correlation of ANXA1 expression with drug resistance and relapse in bladder cancer. International journal of clinical and experimental pathology. 2014; 7:5538-5548.

47. Meng Q, Lei T, Zhang M, Zhao J, Zhao XH, Zhang M. Identification of proteins differentially expressed in adriamycin-resistant (pumc-91/ADM) and parental (pumc-91) human bladder cancer cell lines by proteome analysis. Journal of cancer research and clinical oncology. 2013; 139:509-519. 